Int. J. Elect. Enging. Edhe, Vol. 31, pp. 152 154. Manchester U.P., 1994. Printed in Great Britain

\title{
ON APPROXIMATING SUMS BY MAXIMUMS AND VICE VERSA
}

\author{
HALDUN M. OZAKTAS \\ Department of Electrical Engineering, Bilkent University, Ankara, Turkey
}

We illustrate by way of simple examples the usefulness and instructiveness of the approximation

$$
x+y \approx \max (x, y) \quad x, y \geq 0 .
$$

We will see that this approximation can be useful both ways, i.e. either the left hand or right hand side can be approximated by the other.

For instance, consider the frequency response $|\mathscr{H}(\omega)|=1 / \sqrt{1+\omega^{2} / \omega_{0}^{2}}$. Employing equation $(1)$, we obtain $|\mathscr{H}| \approx 1 / \sqrt{\max \left(1, \omega^{2} / \omega_{0}^{2}\right)}=\min \left(1, \omega_{0} /(1)\right)$. The components of the last expression correspond to the asymptotes of $|\mathscr{H}|$ on a Bode plot (a log-log plot of $\mathscr{H}$ vs. $\omega$ ). Over the greater part of the range of $\omega$ (for which either $\omega \gg \omega_{0}$ or $\omega \ll \omega_{0}$ is satisfied), the approximation is a good one. In the worst case, when $\omega=\omega_{0}$, the error is $3 \mathrm{~dB}$.

Electrical engineering and physical science abound with quantities which vary over several orders of magnitude. It is usually more meaningful to represent such quantities on logarithmic, rather than linear scales, and compare them by looking at their ratios, rather than their differences. Whereas we would say that person $A$ is $10 \mathrm{~cm}$ taller than person $B$, we say that copper conducts electricity 10 times better than lead.

Often in the course of analyzing a physics or engineering problem, the algebra - though conceptually elementary - quickly becomes quite involved, such that an analytical solution is not possible, or is so complicated that its interpretation is difficult. Students are often tempted to carry out the analysis numerically, at the loss of much transparency, understanding and flexibility. In cases where an error by a factor of the order of unity is acceptable, the use of certain approximations can enable a simple analytic solution. Even when greater accuracy is needed, it is still desirable to have an analytic expression which we can use to gain insight into a more exact numerical solution.

As an example, consider the equation $y=a y^{1 / 2}+b$ where all quantities are positive. Although an analytic solution is possible $\left(y=\left(a+\sqrt{\left.\left.a^{2}+4 b\right)^{2} / 4\right)}\right.\right.$, it is much simpler to use equation (1) for the right hand side to obtain $y \approx \max \left(a y^{1 / 2}, b\right)$, which can be solved as* $y \approx \max \left(a^{2}, b\right)$, which can then be expressed as $y=a^{2}+b$, again by using equation (1). Since equation (1) is used

\footnotetext{
"This step is exact, i.e. $y=\max \left(a y^{1 / 2}, b\right)$ has the solution $y=\max \left(a^{2}, b\right)$.
} 
once forwards and once backwards, we are assured that the error is bounded by a factor not exceeding 2, but probably smaller. (For this particular example, the error can be shown to be bound by $4 / 3$ for all values of $a$ and $b$.)

As another example, assume that $\tau$ is governed by the equation $\tau=a N^{m}+\sqrt{b N^{1 / m} / \tau}$, where again all quantities are positive. We desire to minimize the growth rate of $\tau$ as a function of $N$ by choice of $m$ and find the resulting expression for $\tau$. Let us write $\tau \approx \max \left(a N^{m},\left(b N^{1 / m} / \tau\right)^{1 / 2}\right)$, which can be solved as** $\tau \approx \max \left(a N^{m}, b^{1 / 3} N^{1 / 3 m}\right)$. After substitution of the optimal value of $m$ and going back to the summation form, we find $\tau \approx\left(a+b^{1 / 3}\right) N^{1 / \sqrt{3}}$.

Let us now solve the problem of finding the minimum value of $f(x)=$ $g_{1}(x)+g_{2}(x)$ where $g_{1}(x)$ is monotonically increasing and $g_{2}(x)$ is monotonically decreasing. Writing $f^{\prime}(x) \approx \max \left(g_{1}(x), g_{2}(x)\right)$ we see that the minimum value of $f(x)$ is given by $\max \left(g_{1}\left(x_{0}\right), g_{2}\left(x_{0}\right)\right) \approx g_{1}\left(x_{0}\right)+g_{2}\left(x_{0}\right)=2 g_{1}\left(x_{0}\right)$, where $x_{0}$ is the solution of $g_{1}(x)=g_{2}(x)$. The minimum value of $f(x)$ will be determined by this method within a factor of 2 , however it should be noted that the value of $x_{0}$ minimizing $f(x)$ found in the process may be less accurate.

A geometrical interpretation of equation (1) is possible by referring to a right angled triangle with sides $a, b$ and $\sqrt{a^{2}+b^{2}}$, the latter which can be approximated as $\approx \sqrt{\max \left(a^{2}, b^{2}\right)}=\max (a, b)$.

Until now we have illustrated cases where it is beneficial to approximate $x+y$ as $\max (x, y)$. Now we give a few examples of the opposite case. The maximum function arises naturally in engineering problems where the greatest of several causes limits the value of a particular parameter. For instance, the total time it takes to manufacture a certain product with two components is equal to the maximum of the times it takes to manufacture each (perhaps plus the time it takes to assemble them together), assuming the components are worked on simultaneously.

Say that we desire to integrate $\max (f(x), g(x))$ over a specified interval. We may approximate

$$
\begin{aligned}
\int \max (f(x), g(x)) \mathrm{d} x & \approx \int(f(x)+g(x)) \mathrm{d} x=\int f(x) \mathrm{d} x+\int g(x) \mathrm{d} x \\
& \approx \max \left(\int f(x) d x, \int g(x) \mathrm{d} x\right) .
\end{aligned}
$$

Thus the maximum function can be taken out of the integral by incurring an error of at most a factor of 2.

As a more complicated example, we consider the solution of the recursion $L_{i}=\max \left(a_{i}, b_{i}\right)+L_{i-1}$ with $L_{0}$ given. $\left\{a_{i}\right\}$ and $\left\{b_{i}\right\}$ are geometric sequences whose sums can be calculated in closed form easily. Replacing the maximum

\footnotetext{
**Again, this step is exact, i.e. equations of the form $y=\max \left(c, d / y^{n}\right)$ have the solution $y=$ $\max \left(c, d^{1 /(n+1)}\right)$. General equations of the form $f_{0}(y)=\max \left(f_{1}(y), f_{2}(y)\right)$ may be likewise solved in piecewise manner.
} 
function with a summation we obtain $L_{i} \approx a_{i}+b_{i}+L_{i-1}$. Now, assume that $L_{i-1}$ is known from the previous iteration with an error bound by a factor of 2. Since $a_{i}+b_{i}$ equals $\max \left(a_{i}, b_{i}\right)$ within a factor of 2 and since any linear combination of terms with errors bound by a certain factor is also in error by at most that factor, $L_{i}$ is given by this expression within a factor of 2 . That is, the error does not accumulate with each iteration and is always bound by a factor of 2. Thus we find the solution as $L_{N} \approx A+B+L_{0} \approx \max (A, B)+L_{0}$ where

$$
A=\sum_{i=1}^{N} a_{i} \quad \text { and } \quad B=\sum_{i=1}^{N} b_{i} .
$$

It is possible to extend the method described in this paper to sums involving three (or more) terms, but the error in this case will be bound by a factor of 3 . Also, if the minimum, rather than maximum function is involved, it is possible to employ the expression $\min (x, y) \approx 1 /(1 / x+1 / y)$ for $x, y>0$, although this approximation is found to be not as useful as that for the maximum function.

In conclusion, we have illustrated with simple examples the usefulness of the approximation $\max (x, y) \approx x+y$. For more complicated examples better showing the power of this method, the reader is referred to Ref. [1] where such approximations have been extensively used.

I am grateful to Prof. Adolf W. Lohmann of the Physics Institute of the University of Erlangen-Nürnberg, FRG for his suggestions, and also acknowledge the support of the Alexander von Humboldt Foundation.

\section{REFERENCE}

[1] Ozaktas, Haldun M., 'A physical approach to communication limits in computation'. PhD thesis. Stanford University, California, U.S.A. (1991)

\section{ABSTRACTS - ENGLISH, FRENCH, GERMAN, SPANISH}

On approximating sums by maximums and vice versa

We discuss the approximation $\max (x, y) \approx x+y$ for $x, y>0$, which is found to be useful in obtaining simple and transparent approximate solutions and interpretations for analytically complicated problems.

Sur l'approximation de sommes par maxima et vice-versa

Cet article discute l'approximation $\max (x, y) \approx x+y$ pour $x, y>0$, qui est utile pour obtenir des solutions approchées simples et transparentes et permet d'interpréter des problèmes analytiques compliqués.

\section{Uber die Annäherungssummen durch Höchstbeträge und umgekehrt}

Wir besprechen die Annäherung $\max (x, y) \approx x+y$ für $x, y>0$, die sich als nützlich erweist, um einfache und transparente annähernde Lösungen und Interpretationen für analytisch komplizierte Probleme zu erhalten.

\section{Aproximando sumas por máximos y viceversa}

Se discute la aproximación max $(x, y) \approx x+y$ para $x, y>0$, la cual resulta útil en la obtención de interpolaciones y soluciones simples y transparentes para problemas analíticos complejos. 